\title{
Tamdy Virus in Ixodid Ticks Infesting Bactrian Camels, Xinjiang, China, 2018
}

\section{Hong Zhou, ${ }^{1}$ Zhenghai Ma, ${ }^{1}$ Tao Hu, Yuhai Bi, Amutikari Mamuti, Runyuan Yu, Michael J. Carr, Mang Shi, Juan Li, Kirill Sharshov, George F. Gao, Weifeng Shi}

Author affiliations: Shandong First Medical University \& Shandong Academy of Medical Sciences, Taian, China (H. Zhou, T. Hu, R. Yu, J. Li, W. Shi); Xinjiang University, Urumqi, China (Z. Ma, A. Mamuti); Chinese Academy of Sciences, Beijing, China (Y. Bi, G.F. Gao); University College Dublin, Dublin, Ireland (M.J. Carr); Hokkaido University, Sapporo, Japan (M.J. Carr); Sun Yat-sen University, Guangzhou, China (M. Shi); The University of Sydney, Sydney, New South Wales, Australia (M. Shi); Research Institute of Experimental and Clinical Medicine, Novosibirsk, Russia (K. Sharshov); Chinese Center for Disease Control and Prevention, Beijing (G.F. Gao)

DOI: https://doi.org/10.3201/eid2511.190512

We isolated Tamdy virus (TAMV; strain XJ01/TAMV/China/2018) from Hyalomma asiaticum ticks infesting Bactrian camels in Xinjiang, China, in 2018. The genome of the strain showed high nucleotide similarity with previously described TAMV strains from Asia. Our study highlights the potential threat of TAMV to public health in China.

$\mathrm{T}$ amdy virus (TAMV) was first isolated from the tick species Hyalomma asiaticum asiaticum collected from sheep in the Tamdinsky district of the Bukhara region, Uzbekistan, in 1971 (1). Subsequently, large-scale surveillance of TAMV from Ixodidae ticks using newborn mice successfully isolated 47 TAMV strains from various tick species from Armenia, Kazakhstan, Kyrgyzstan, Turkmenistan, and Uzbekistan, highlighting both its widespread distribution and its ability to infect mammals (2). Recently, TAMV was identified in Turkey from Hyalomma spp. ticks collected from Meriones tristrami gerbils in the Middle East (3). Sequence comparison and phylogenetic analyses of full-length TAMV genomes from different subspecies of $H$. asiaticum ticks taxonomically classified it in the genus Nairovirus, family Bunyaviridae (4). TAMV was also detected in 1973 from a febrile patient in Kyrgyzstan (5).

In May 2018, fourteen ticks attached to 2-humped Bactrian camels (Camelus bactrianus) were collected from a camel farm in Xinjiang, China. We extracted total RNA of the ticks using the E.Z.N.A. Total RNA Kit (Omega Bio-tek, https://www.omegabiotek.com). We used a

${ }^{1}$ These authors contributed equally to this article. transcriptomics approach to investigate the viruses harbored by the ticks and used the BGI mRNA Library Preparation protocol according to MGIEasy mRNA Library Prep Kit (BGI, https://www.bgi.com) to construct the RNA sequencing libraries for each tick. We conducted paired-end (100-bp) sequencing of each RNA library on the BGISEQ500RS platform (BGI). We obtained 493,090,699 raw reads and then adaptor and quality trimmed them with the Fastp program (6), resulting in a total of 492,344,756 clean reads. These reads were de novo assembled using Trinity (7) with default settings. We compared the assembled contigs using BLASTn (http://blast.ncbi.nlm.nih. gov/blast.cg) against the nucleotide database downloaded from GenBank, with an E-value cutoff set at $1 \times 10^{-5}$.

We identified contigs annotated as the large (L), medium (M), and small (S) gene segments of TAMV (family Nairoviridae, genus Orthonairovirus) in 1 tick (pool 10). To confirm the assembled viral contigs, we mapped reads back to the full-length genome of the TAMV strain LEIV-1308Uz (GenBank accession nos. KP792726-8, corresponding to the $\mathrm{L}, \mathrm{M}$, and $\mathrm{S}$ gene segments) as reference with Bowtie2 (8) and inspected using Geneious version 11.1.5 (Biomatters, Ltd., https://www.geneious.com). After removing repetitive reads, we mapped 34,172 reads to the L gene segment (depth $252 \pm$ SD 120), 60,184 reads to the M gene segment (depth: $1186 \pm 418$ ), and 8,724 reads to the S gene segment (depth $392 \pm 138$ ). The virus genome obtained comprised L segment (encoding the RNA-dependent RNA polymerase [RdRp]), 12,215 bp; M segment (encoding the glycoprotein precursor), 4,565 bp; and $\mathrm{S}$ segment (encoding the nucleocapsid), 2,005 bp.

We then cultured the grinding fluid supernatant corresponding to pool 10 in Vero cells in Dulbecco Modified Eagle medium. We observed apparent cytopathic effects, such as higher cell refractive index, cell shrinkage, size reduction, rounding, and shedding, in infected Vero cells at 11 days postinfection (Figure, panel A). The virus strain was named XJ01/TAMV/China/2018 (hereafter XJ01). After 2 passages, we performed further transcriptome sequencing of the first- and second-generation virus suspension from cell cultures. We assembled the complete genome sequences of XJ01 again, as described, and found that the TAMV genomes from cell cultures were identical to those from the original sample.

To confirm the genome sequence of XJ01, we designed 14 paired primers for Sanger sequencing (Appendix Table, https://wwwnc.cdc.gov/EID/article/25/11/19-0512-App1. pdf). The consensus gene sequences of Sanger sequencing of the amplified products were consistent with those from transcriptome sequencing and were deposited in GenBank (accession nos. MK757580-2). Sequence comparison revealed that XJ01 was highly similar to 3 previously described TAMV strains from Asia; sequence identities were 
94.8\%-94.9\% for the L segment, $93.5 \%-94.7 \%$ for the M segment, and $95.4 \%-96.8 \%$ for the $\mathrm{S}$ segment.

Phylogenetic analysis of representative strains of the family Nairoviridae using RAxML (9) revealed that the 4

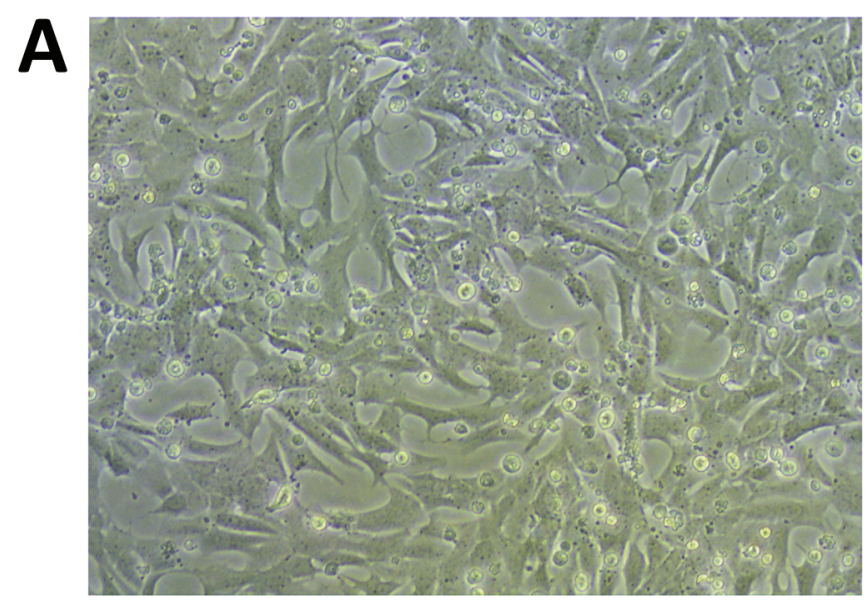

TAMV strains clustered with high bootstrap support and fell within the Orthonairovirus genus in the RdRp tree (Figure, panel B). In addition, they were closely related to several other orthonairoviruses from ticks, including Burana virus,

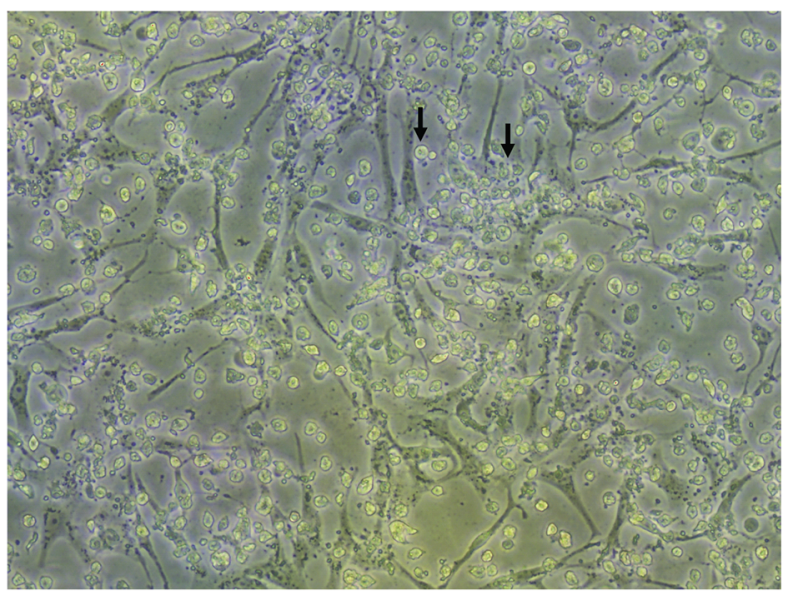

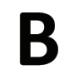

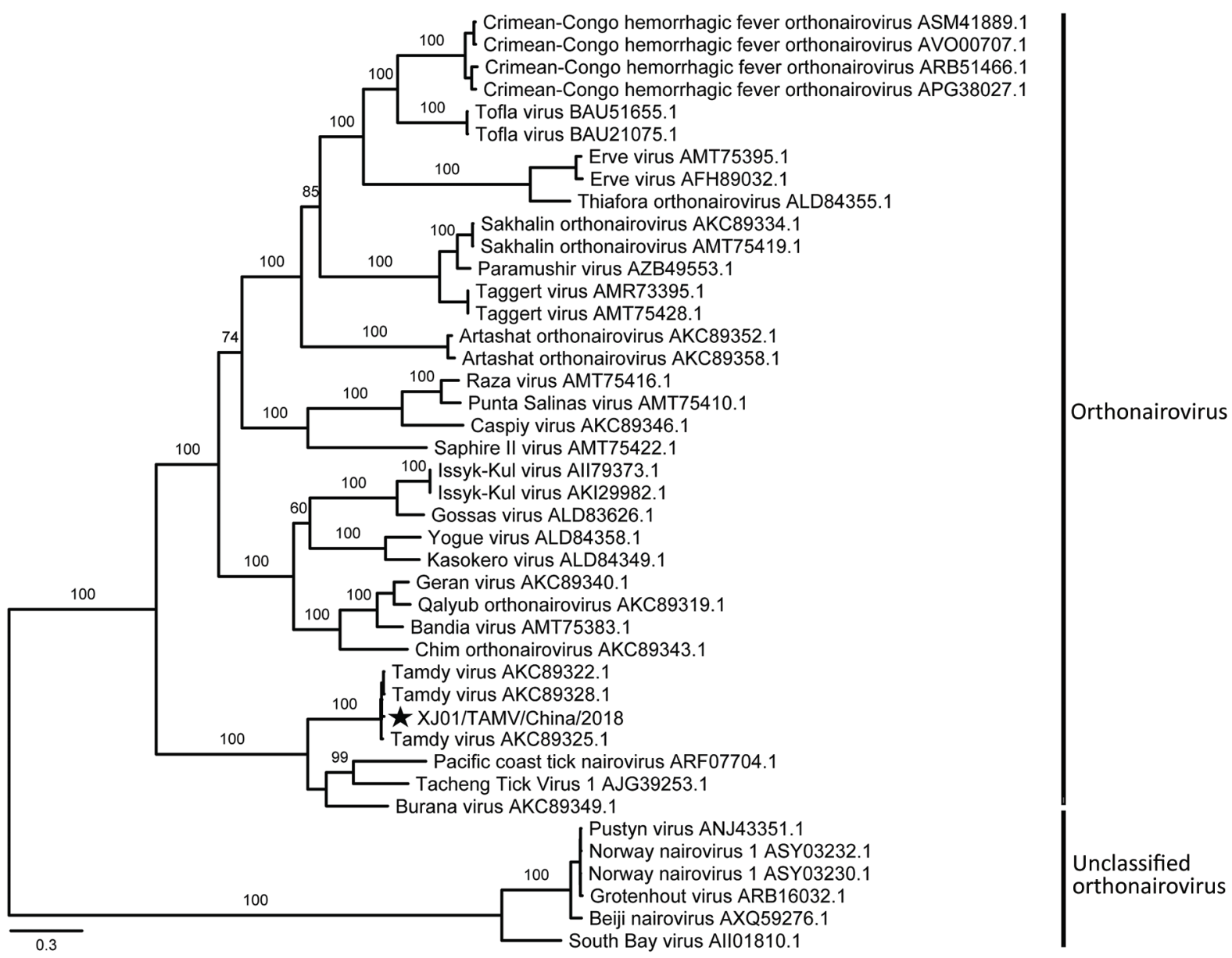

Figure. Identification of the Tamdy virus (TAMV) strain XJ01/TAMV/China/2018 from Hyalomma asiaticum ticks infesting Bactrian camels in Xinjiang, China, 2018, by cell culture and phylogenetic analysis. A) Light micrographs of cytopathic effects caused by TAMV infection at 11 days postinfection. Left, normal Vero cells as control; right, infected Vero cells with apparent cytopathic effects (black arrows). Original magnification $\times 100$. B) Phylogenetic analysis of the RNA-dependent RNA polymerase protein sequences of TAMV and representative viruses in the family Nairoviridae. Scale bar indicates nucleotide substitutions per site. Star indicates strain from this study. 
Tacheng tick virus 1, and Pacific coast tick nairovirus in all the RdRp, glycoprotein precursor, and nucleocapsid trees and formed a small cluster in the Orthonairovirus genus (Figure, panel B; Appendix Figures 1, 2).

We also obtained the cytochrome c oxidase gene sequence of the tick and deposited it in GenBank (accession no. MK757583). A BLASTn search (https://blast.ncbi.nlm. nih.gov/Blast.cgi) revealed that the top hit was from $H$. asiaticum (GenBank accession no. KX882103.1) with sequence identity of $99 \%$; this species is a widely distributed tick in China, especially in northwestern China (10).

In summary, we identified a TAMV strain from Ixodid ticks collected in Xinjiang, China, that poses a threat to public health in Xinjiang and even globally. Because of the ability of TAMV to infect mammals including humans, the lack of effective antiviral drugs and prophylactic vaccines, and the widespread distribution of its major host in China, extensive TAMV surveillance is urgently needed.

This study was supported by the National Science and Technology Major Project (2018ZX10101004-002), the National Key Science and Technology Projects of China (2017ZX10104001-006), the Academic Promotion Plan of Shandong First Medical University \& Shandong Academy of Medical Sciences, and the Strategic Priority Research Program of the Chinese Academy of Sciences (XDB29010102). W.S. was supported by the Taishan Scholars program of Shandong Province (ts201511056).

\section{About the Author}

Dr. Zhou obtained her PhD in microbiology at Shandong University and now is a lecturer at Shandong First Medical University. Her research interests include viromics and novel virus discovery.

\section{References}

1. Lvov DK, Sidorova GA, Gromashevsky VL, Kurbanov M, Skvoztsova LM, Gofman YP, et al. Virus "Tamdy"-a new arbovirus, isolated in the Uzbee S.S.R. and Turkmen S.S.R. from ticks Hyalomma asiaticum asiaticum Schulee et Schlottke, 1929, and Hyalomma plumbeum plumbeum Panzer, 1796. Arch Virol. 1976;51:15-21. PubMed https://doi.org/10.1007/BF01317830

2. L'vov DK, Sidorova GA, Gromashevskiǐ VL, Skvortsova TM, Aristova VA. Isolation of Tamdy virus (Bunyaviridae) pathogenic for man from natural sources in Central Asia, Kazakhstan and Transcaucasia [in Russian]. Vopr Virusol. 1984;29:487-90.

3. Brinkmann A, Dinçer E, Polat C, Hekimoğlu O, Hacıoğlu S, Földes K, et al. A metagenomic survey identifies Tamdy orthonairovirus as well as divergent phlebo-, rhabdo-, chu- and flavi-like viruses in Anatolia, Turkey. Ticks Tick Borne Dis. 2018;9:1173-83. https://doi.org/10.1016/j.ttbdis.2018.04.017

4. L'vov DK, Al'khovskiı̌ SV, Shchelkanov MI, Shchetinin AM, Aristova VA, Gitel'man AK, et al. Taxonomy of previously unclassified Tamdy virus (TAMV) (Bunyaviridae, Nairovirus) isolated from the Hyalomma asiaticum asiaticum Schülce et Schlottke, 1929 (Ixodidae, Hyalomminae) in the Middle East and transcaucasia [in Russian]. Vopr Virusol. 2014;59:15-22.

5. Karas FR, Vargina SG, Steblyanko SN, Kolpakov VN, Seropolko AA. Ecology of Tamdy virus in Kyrgystan. Proceedings of X Symposium. Ecology of viruses. Baku (Azerbaijan): Ministry of Health of Azerbaijan Republic of USSR; 1976. p. 87-88.

6. Chen S, Zhou Y, Chen Y, Gu J. fastp: an ultra-fast all-in-one FASTQ preprocessor. Bioinformatics. 2018;34:i884-90. https://doi.org/10.1093/bioinformatics/bty560

7. Grabherr MG, Haas BJ, Yassour M, Levin JZ, Thompson DA, Amit I, et al. Full-length transcriptome assembly from RNA-Seq data without a reference genome. Nat Biotechnol. 2011;29:644-52. https://doi.org/10.1038/nbt.1883

8. Langmead B, Salzberg SL. Fast gapped-read alignment with Bowtie 2. Nat Methods. 2012;9:357-9. PubMed https://doi.org/ 10.1038/nmeth. 1923

9. Stamatakis A, Ludwig T, Meier H. RAxML-III: a fast program for maximum likelihood-based inference of large phylogenetic trees. Bioinformatics. 2005;21:456-63. PubMed https://doi.org/10.1093/ bioinformatics/bti191

10. Sheng J, Jiang M, Yang M, Bo X, Zhao S, Zhang Y, et al. Tick distribution in border regions of northwestern China. Ticks Tick Borne Dis. 2019;10:665-9.

Address for correspondence: Weifeng Shi, Shandong First Medical University \& Shandong Academy of Medical Sciences, Key Laboratory of Etiology and Epidemiology of Emerging Infectious Diseases in Universities of Shandong, Yingshengdonglu 2, Taian 271000, Shandong, China; email: shiwf@ioz.ac.cn,wfshi@sdfmu.edu.cn

\section{Introduction of Avian Influenza A(H6N5) Virus into Asia from North America by Wild Birds}

\section{Sol Jeong, Dong-Hun Lee, Yu-Jin Kim, Sun-Hak Lee, Andrew Y. Cho, Jin-Yong Noh, ${ }^{1}$ Erdene-Ochir Tseren-Ochir, ${ }^{2}$ Jei-Hyun Jeong, Chang-Seon Song}

Author affiliations: University of Connecticut, Storrs, Connecticut, USA (S. Jeong, D.-H. Lee); Konkuk University, Seoul, South Korea (S. Jeong, Y.-J. Kim, S.-H. Lee, A.Y. Cho, J.-Y. Noh, E.-O. Tseren-Ochir, J.-H. Jeong, C.-S. Song)

DOI: https://doi.org/10.3201/eid2511.190604

${ }^{1}$ Current affiliation: Konkuk Ctc bio Animal Vaccine (KCAV) Co., Ltd., Seoul, South Korea.

${ }^{2}$ Current affiliation: Mongolian University of Life Sciences, Ulaanbaatar, Mongolia. 